\title{
Current status of anorexia nervosa treatment
}

\author{
Aslı Melike Ekmekçi ${ }^{1}$ (D), Oytun Erbaş² \\ ${ }^{1}$ Institute of Experimental Medicine, Gebze-Kocaeli, Turkey \\ ${ }^{2}$ Department of Physiology, Medical Faculty of Demiroğlu Bilim University, Istanbul, Turkey
}

\begin{abstract}
Anorexia nervosa (AN) is an eating disorder characterized by low body weight as a result of restrictive eating behaviors. Although it is mostly seen in adolescent women, it can also occur in adulthood. Diets in which energy intake is restricted, heavy sports and obsessive movements were evaluated as distinct symptoms of AN. Anorexia nervosa has the highest mortality rate among psychiatric disorders. Although there are alternative approaches for treatment, there is no clear application. Genetic and neurobiological mechanisms of AN were examined for a specific treatment. Dietitiancontrolled diet was evaluated as an effective method for weight gain for AN treatment. Family-based treatment and adolescent-focused therapy in adolescents and Maudsley model applications in adults were examined for conditions with psychiatric comorbidity. What the drug treatments were and at what dose they should be applied in AN were evaluated. Finally, promising molecules in AN treatment were mentioned. It has been observed that an interdisciplinary approach is more curative in the treatment of AN. This review is a synthesis of literature studies on the definition, diagnosis, and treatment of anorexia nervosa.

Keywords: Anorexia nervosa, diagnosis, nutrition, treatment.
\end{abstract}

Anorexia nervosa (AN) is an eating disorder, prevalent especially among adolescent women, characterized by restriction of energy intake through self-imposed weight-loss. Anorexia nervosa has the highest mortality among psychiatric disorders. Average age of onset is between ages 15-19, which is a critical period in terms of development. Anorexia nervosa prevalence is generally highest in western countries. Lifetime prevalence among the general population is $1 \%$ in women and less than $0.5 \%$ in men. ${ }^{[1,2]}$ Due to excessive fear of gaining weight, patients with AN exhibit behavior such as self-induced vomiting and abuse of diuretics. They may constantly obsess over their body appearance. They are physically active and exercise obsessively. ${ }^{[3-5]}$ No definite treatment of AN has been determined. Pharmacologic alternatives and seemingly effective psychotherapy and nutritional interventions may be applied. It is thought that an approach with a multidisciplinary team may be more effective. Therefore, it is anticipated that treatment is managed by a team comprised of pediatric/adolescent psychiatrists, dieticians, physiotherapists, and experienced nurses. ${ }^{[5]}$

\section{IDENTIFICATION AND DIAGNOSIS}

Anorexia nervosa is usually noticed by the individual's parents or friends before doctor diagnosis. Primary symptoms include low body mass index (BMI) as a result of restricted energy intake, distorted perception of body image, weight gain phobia, endocrine disorders, amenorrhea in women, loss of sexual interest and strength in men, obsessive behaviors, anxiety, and depression. While BMI is below $18.5 \mathrm{~kg} / \mathrm{m}^{2}$ at initial onset, it has been observed to fall below $15 \mathrm{~kg} / \mathrm{m}^{2}$ in severely malnourished cases. ${ }^{[6,7]}$ 
Psychiatric and Physiological Comorbidity: Anorexia nervosa negatively affects physical, neurological, and psychiatric development. In a study of 211 adolescents, it was observed that mean height of AN patients was significantly shorter than the normal adolescent group. ${ }^{[8]}$ Patients who were treated before the start of pubertal growth spurt showed a better growth curve than those who began treatment after growth spurt had started. ${ }^{[9]}$ Early diagnosis and treatment are believed to be effective in preventing developmental disorders. The studies observed that risk of amenorrhea persisted in patients with premenarchal onset of AN despite weight gain. ${ }^{[10]}$

Increased prevalence of autoimmune diseases has been observed in AN, mostly type 1 diabetes. Type 1 diabetes usually emerges before AN. ${ }^{[2]}$ One nationwide study conducted in Sweden established a bidirectional relationship between AN-celiac disease and AN-Crohn's disease. ${ }^{[11]}$ Decreased levels of sexual steroid hormones lead to brain development disorders. ${ }^{[12]}$ There are no studies on whether or not there is permanent neurological damage after treatment in adolescent AN patients. Apart from these, depression, dysthymia, anxiety, and obsessive-compulsive disorder (OCD) are reported as common mental disorders in AN. ${ }^{[13]}$ According to one study, $26 \%$ of 31 female patients who were diagnosed with OCD had symptoms of AN. ${ }^{[14]}$ Increased symptoms of autism spectrum disorder (ASD) have been reported in AN patient groups. Studies have shown high rates of AN among children and adolescents with severe autism. ${ }^{[15]}$ Until the gender differences of ASD

Table 1. Complications seen in anorexia nervosa ${ }^{[5]}$

\begin{tabular}{|c|c|}
\hline Cardiologic & $\begin{array}{l}\text { Bradycardia, hypotension, mitral valve } \\
\text { prolapse, pericardial effusion }\end{array}$ \\
\hline Structural & $\begin{array}{l}\text { Low body mass index, muscle wasting, hypo- } \\
\text { thermia }\end{array}$ \\
\hline Dermatologic & Carotenoderma, lanugo, xerosis \\
\hline Endocrine & $\begin{array}{l}\text { Hypothalamic hypogonadism, osteopenia, } \\
\text { osteoporosis }\end{array}$ \\
\hline Gastrointestinal & Constipation, pancreatitis \\
\hline Hematologic & Cytopenia, bone marrow hypoplasia/aplasia \\
\hline Neurologic & $\begin{array}{l}\text { Brain atrophy, peripheral neuropathy } \\
\text { (mineral and vitamin deficiencies) }\end{array}$ \\
\hline Psychiatric & $\begin{array}{l}\text { Obsessive-compulsive disorder, anxiety } \\
\text { disorder, depression }\end{array}$ \\
\hline
\end{tabular}

are understood, it is thought that ASD diagnosis will remain problematic in patients with $A N$ associated with comorbidity. Since psychiatric comorbidities are fairly common among patients with AN, effective psychotherapeutic treatments are needed (Table 1). ${ }^{[16]}$

\section{PATHOGENESIS}

Genetics: Multiple factors have been identified in the pathology of AN. We believe these systems must be well understood to ensure definitive treatment. It has been stated that genetic predisposition is necessary but insufficient alone for the occurrence of AN. ${ }^{[17]} \mathrm{A}$ genomewide association study of about 17,000 patients with AN and 55,000 healthy controls identified eight significant independent genetic loci. So far, approximately 130 polymorphisms related to eating attitudes, regulation of eating behavior, motivation, and rewards have been identified in 43 genes. Scientists have placed great emphasis on genetic factors of centers that control food intake, such as agouti-associated peptide, opioid receptor delta-1, or brain-derived neurotrophic factor-related polymorphisms. ${ }^{[18]}$ Further studies are required to understand the effect of genetic predisposition in AN.

Neurobiology: Another substance that has an important effect on the regulation of food intake is orexins. Orexin-A (OXA) is a 33 amino acid orexigenic neuropeptide responsible for sleep-wakefulness and is involved in the regulation of eating behavior and attitude. ${ }^{[19]}$ In those at ideal body weight, hunger or restriction of food intake causes an increase in serum OXA which normalizes with return to nutrition. Differences in OXA concentration are unclear in patients with AN. Studies have shown both an increase and decrease in OXA levels in plasma. ${ }^{[20]}$ This indicates that OXA concentrations are not associated with body composition (body fat reserves) or BMI, and that its release can only be regulated by energy status. Studies have shown that OXA concentration does not directly affect the outcome, but indirectly through the regulation of sleep and wakefulness in AN. ${ }^{[19,21]}$ Reduced food intake in AN has been shown to increase the activation of opioids in the brain. This is thought to lead to the continuation of the restrictive diet, but triggering positive thoughts. Impaired dopaminergic and serotonergic transmission 
have been identified in AN. Increased dopamine D2 and D3 receptor binding in the anterior ventral striatum, as well as intensified 5-HT1A and decreased 5-HT2A receptor binding have been reported. These abnormalities have been associated with emotional reactions that affect patients' eating behaviors. Normally, eating a delicious meal causes dopamine release in the striatum, however, people with AN experience intense anxiety rather than positive emotions during endogenous dopamine release. This may explain how they maintain their restrictive diet. ${ }^{[22]}$

Clearly learning when behavior such as restrictive food intake has become a habit or which nerve pathways are effective may provide hope for recovery. ${ }^{[23]}$

\section{TREATMENT METHODS}

\section{What effects recovery and what is the prognosis?}

It has been observed that remission in AN is varying. A portion of outpatients have been reported to recover in a shorter period of time. Relapse was found more common among patients with psychiatric comorbidity and long-term AN. Comorbidity is associated with poor prognosis. Recently, it was demonstrated that complete recovery was possible even in severe, chronic AN of 21 years. ${ }^{[17,24]}$ The patient's cardiovascular and immune system, fertility, and bone density may return to normal when the patient is protected against damage from refeeding and acute disease progression. Since bone recovery occurs over a long period of time, the spine and pelvis must be protected against physical activity, especially after weight gain. It has been observed that psychological recovery is more difficult, even if the patient gains motivation to gain weight or overcome the values imposed by being underweight. ${ }^{[25]}$

\section{Is pharmacologic treatment effective?}

There is weak evidence regarding drug use in AN. Although antidepressants are usually used to treat depressive symptoms, they have shown limited success. Olanzapine and risperidone are commonly used as antipsychotics for a short duration in first step treatment. The use of selective serotonin reuptake inhibitors (SSRI) to prevent relapse and bupropion in cases of increased seizure risk have been mentioned in the literature, though its benefits are unclear. ${ }^{[26-28]}$ One randomized study evaluated 152 AN patients undergoing outpatient treatment, in which 75 received olanzapine and 22 received placebo. Olanzapine was initiated at $2.5 \mathrm{mg} /$ day (1 pill daily) dosage which was increased to $5 \mathrm{mg} /$ day (2 pills daily) in two weeks. On the fourth week, the drug was increased to maximum dosage of $10 \mathrm{mg} /$ day (4 pills daily). At the end of the study, although weight gain was observed compared to placebo, there were no significant benefits in terms of psychological symptoms. ${ }^{[29]}$ As there are limited studies on pharmacological treatments, further comprehensive evidence-based studies are needed.

\section{What is the role of psychotherapy?}

Cognitive behavioral therapy and interpersonal psychotherapy that are effective in other eating disorders are not definitive solutions for AN. Although psychotherapeutic interventions are a preferred method in AN, the outcome of psychotherapy varies depending on the stage of the disease. While the response to psychological treatment was quite good in adolescents with shortterm AN, it was observed that adults with a more severe course of the disease responded poorly to treatment. In addition, it was observed that adults had high rates of discontinuing treatment. ${ }^{[30]}$ One report found no difference between behavioral therapy and cognitive therapy. ${ }^{[31]}$ According to small studies, cognitive-behavioral therapy (CBT), interpersonal therapy (IPT), cognitive analytical therapy, and family therapy have been studied, but none have been found to be better in terms of effectiveness. ${ }^{[32]}$

Family based treatment (FBT) consists of three phases. ${ }^{[33]}$ In the first phase, parents are motivated to take responsibility for the cause of illness and address the positive aspects of their parenting. Families are encouraged on how to best help their child gain weight. In the second phase, parents are assisted in how they can properly apply eating and weight management. The third phase focuses on how parents can form a healthy relationship with their adolescent. ${ }^{[34]}$

Adolescent-focused therapy (AFT) posits that individuals with AN self-control with biological needs due to lack of ego. Patients learn to tolerate their affective states rather than starving themselves. In Phase 1, the therapist assesses 
motivation by establishing relationships and recognizing the patient's psychological concerns. By emphasizing changing restrictive behaviors and setting goals, the therapist encourages the patient to stop dieting and to gain weight. Phase 2 focuses on developing the ability to tolerate negative emotions. Phase 3 involves termination. Adolescent-focused therapy sessions are applied for 45 minutes as 32 sessions throughout the treatment year. To increase the functionality of parents and inform them of progress, parents are included in this treatment model by holding meetings where only the parents are present. ${ }^{[35,36]}$

For adults with AN, Maudsley (MANTRA) treatment suggests that some factors associated with obsessive and anxious personality traits are central in AN and should be addressed in treatment. It is applied in the form of motivational interview. By performing an in-depth evaluation of physical, psychological, neuropsychological, and socio-emotional relationships, a treatment strategy is developed based on the patient's strengths. The individual's clinical profile, treatment motivation, and level of medical risk is assessed and treatment is planned.

MANTRA treatment proposes to improve clinical outcomes (symptoms of eating disorder) by addressing factors including thinking style characterized by fear of making mistakes, disorders in the socio-emotional domain, pro-anorexia beliefs ("AN keeps me safe, AN numbs my emotions, AN helps me express my problems"), anxiety, blame, and criticism. ${ }^{[37-40]}$

\section{Nutrition Interventions in Anorexia Nervosa}

Nutritional rehabilitation is the most important step in AN treatment. The main objectives of nutritional intervention are to return body weight to normal, to correct the physical complications of malnutrition, to normalize eating habits, and to educate patients about proper and healthy eating habits by improving their motivation. Dieticians have an important role in education. Most of the behavioral changes (food anxiety, taste changes, binge eating, depression, obsessions, apathy, irritability) seen in malnourished AN patients improved with weight gain. Nutrition rate and nutrition treatment vary depending on the setting (outpatient treatment, day hospital, inpatient treatment). It has been observed that regulation of body weight, improvement in menstruation and ovulation in women, normalized sexual desire and hormone levels in men, and improved growth and sexual development in children and adolescents have been observed until normal body weight is achieved. Many studies have been published on nutritional therapy in AN. Some have adopted the "start slow, go slow" approach, meaning a slow re-feeding rate, especially in hospitalized patients with very low BMI. On the other hand, some studies have followed the "start higher, go faster" approach, for a more aggressive re-feeding process with additional phosphate supplements to treat moderately malnourished patients. In general, the results of these studies have shown that re-feeding is safe and effective if adequately supervised and a specific protocol is followed. ${ }^{[41,42]}$ To prevent re-feeding in $\mathrm{AN}$, it was found that 0.5-1.4 kg/week weight gain in hospitalized inpatients, and 250-500 g/week in outpatients was appropriate. ${ }^{[43,44]}$ Although weight loss and malnutrition vary depending on the duration of disease, age of the patient, comorbidity and severity of behavior, weight gain rates have been suggested as 500-1400 g/week. For the first three days of nutrition in hospitalized patients, prophylactic electrolyte supplementation ( $\mathrm{P}, \mathrm{K}, \mathrm{Mg}$ ), thiamine (200-300 mg/day), vitamins (200\% RDI), minerals and trace elements (100\% $\mathrm{RDI}$ ), and sodium limited to $<1 \mathrm{mmol} / \mathrm{kg} /$ day is applied. Adequate monitoring of glucose and electrolyte levels is recommended. ${ }^{[45]}$

According to most protocols, oral nutritional supplements (ONS) are prescribed if patients' nutritional needs are not met or their weight stagnates. However, study groups have sometimes used ONS from the first day of admission to shorten length of hospital stay. Generally, hypercaloric polymeric diets with a caloric density of $1.5 \mathrm{kcal} / \mathrm{mL}$ or higher have been used. $^{[46]}$ In patients with severe eating disorder who reject eating, tube feeding (TF) has become widespread. ${ }^{[4]}$ Polymeric, lactose-free, fiber-rich diets are generally used. Percutaneous endoscopic gastrostomy (PEG) is a limited refeeding option, but may be necessary in patients who require long-term therapy. Parenteral nutrition (PN) has been used even less in AN patients, but has become an alternative in patients with severe nutritional deficiency (digestive bleeding, intestinal 
obstruction, intestinal perforation, ileus, etc.) where the digestive system is not functional. ${ }^{[48,49]}$

\section{Promising Studies in the Treatment of Anorexia Nervosa}

In recent years, researchers have identified several new molecules that may be important for AN therapy. These include neuropeptide $\mathrm{W}(\mathrm{NPW})$, neuropeptide $\mathrm{B}(\mathrm{NPB})$, and vaspin, however, their precise roles and contributions to this disorder are not fully understood as they mainly involve animal studies. Neuropeptide W is an endogenous ligand that belongs to the GPCR receptor family (G-protein-coupled receptors) and contributes to energy homeostasis, regulation of the hypothalamic-pituitary-adrenal axis and secretion. ${ }^{[50]}$ Relatively large amounts of mRNA of the NPW gene have been detected in the human brain, hippocampus, substantia nigra, stomach, and rectum. ${ }^{[51]}$ It was found that its expression in the stomach depends on the level of nutrition and the type of food consumed. ${ }^{[52]}$ High-dose NPW injections ( $3 \mathrm{nmoL} / 100 \mathrm{~g}$ body mass) caused a short-term decrease in leptin and insulin levels in rat blood. However, no changes were observed as a result of administering NPB, a polypeptide with a structure similar to NPW. ${ }^{[53]}$ There are no data regarding NPW and NPB levels in individuals with AN. It has been suggested that vaspin (serine protease inhibitor) may be an antagonist of some yet unknown molecules that reduce insulin activity. Studies have shown that AN patients also have higher levels of this protein in their blood compared to people with normal body mass. A negative correlation was found between serum vaspin level and body mass in individuals with $A N$ and in the control group without this disorder.

Serum oxytocin concentration was about half the amount compared to the age-matched control group. Recently, one study reported that nasally administered oxytocin may be useful in the treatment of anorexia. ${ }^{[54]}$ The opinion that coordinated effort of various medical and psychiatry departments that usually don't work together is dominant in the literature. Due to the affected age group and the relevant time frame, patient care often goes through many processes. We believe that nutritional therapy in collaboration with a dietician provides the most effective recovery and weight gain. Psychotherapies and medications can provide treatment in AN patients with comorbidity. However, we see that a better understanding of the disease's underlying mechanism and more comprehensive studies are needed for a definitive treatment.

In this review study, the diagnostic criteria of AN, characterized by restrictive eating behaviors, are described and treatment methods are emphasized. Studies in the literature were reviewed and it was found that there was no definitive treatment method. In light of the studies investigated in this review, we believe that weight gain best occurs with dietician management and a multidisciplinary approach. Aside from the nutritional approach, various psychotherapies are applied, especially in patients with comorbidity. We believe that family based treatment and adolescent-focused therapy can be applied in addition to nutritional therapy in adolescent patients. Although psychotherapies have exhibited better results in adolescents, the availability of only MANTRA therapy for adults is insufficient and alternative therapeutic approaches are needed. Although the use of olanzapine in drug treatments is more widespread, the definite application is uncertain. In order to form a combination treatment or definitive treatment, we believe that future studies should investigate the answers to questions such as which stage, how much, to whom, at what dose, or for how long the drug should be administered.

\section{Declaration of conflicting interests}

The authors declared no conflicts of interest with respect to the authorship and/or publication of this article.

\section{Funding}

The authors received no financial support for the research and/or authorship of this article.

\section{REFERENCES}

1. Brockmeyer T, Friederich HC, Schmidt U. Advances in the treatment of anorexia nervosa: a review of established and emerging interventions. Psychol Med 2018;48:1228-56.

2. Zipfel S, Giel KE, Bulik CM, Hay P, Schmidt U. Anorexia nervosa: aetiology, assessment, and treatment. Lancet Psychiatry 2015;2:1099-111.

3. Jaite C, van Noort BM, Vloet TD, Graf E, Kappel $\mathrm{V}$, Geissler $\mathrm{J}$, et al. A multicentre randomized controlled trial on trans-generational attention deficit/hyperactivity disorder (ADHD) in mothers and children (AIMAC): an exploratory analysis of 
predictors and moderators of treatment outcome. Z Kinder Jugendpsychiatr Psychother 2019;47:49-65.

4. Kwok C, Kwok V, Lee HY, Tan SM. Clinical and sociodemographic features in childhood vs adolescentonset anorexia nervosa in an Asian population. Eat Weight Disord 2020;25:821-6.

5. Herpertz-Dahlmann B, Dahmen B. Children in Need-Diagnostics, Epidemiology, Treatment and Outcome of Early Onset Anorexia Nervosa. Nutrients 2019;11:1932.

6. Garber AK, Sawyer SM, Golden NH, Guarda AS, Katzman DK, Kohn MR, et al. A systematic review of approaches to refeeding in patients with anorexia nervosa. Int J Eat Disord 2016;49:293-310.

7. American Psychiatric Association. Diagnostic and statistical manual of mental disorders (DSM-5 ${ }^{\circledR}$ ). American Psychiatric Pub.; 2013.

8. Modan-Moses D, Yaroslavsky A, Kochavi B, Toledano A, Segev S, Balawi F, et al. Linear growth and final height characteristics in adolescent females with anorexia nervosa. PLoS One 2012;7:e45504.

9. Swenne I. Poor catch-up growth in late adolescent boys with eating disorders, weight loss and stunting of growth. Eur Eat Disord Rev 2013;21:395-8.

10. Dempfle A, Herpertz-Dahlmann B, Timmesfeld N, Schwarte R, Egberts KM, Pfeiffer E, et al. Predictors of the resumption of menses in adolescent anorexia nervosa. BMC Psychiatry 2013;13:308.

11. Hedman A, Breithaupt L, Hübel C, Thornton LM, Tillander A, Norring C, et al. Bidirectional relationship between eating disorders and autoimmune diseases. $\mathrm{J}$ Child Psychol Psychiatry 2019;60:803-12.

12. Mainz V, Schulte-Rüther M, Fink GR, HerpertzDahlmann B, Konrad K. Structural brain abnormalities in adolescent anorexia nervosa before and after weight recovery and associated hormonal changes. Psychosom Med 2012;74:574-82.

13. Pinhas L, Morris A, Crosby RD, Katzman DK. Incidence and age-specific presentation of restrictive eating disorders in children: a Canadian Paediatric Surveillance Program study. Arch Pediatr Adolesc Med 2011;165:895-9.

14. Tamburrino MB, Kaufman R, Hertzer J. Eating disorder history in women with obsessive compulsive disorder. J Am Med Womens Assoc (1972) 1994;49:24-6.

15. Margari L, Palumbi R, Peschechera A, Craig F, de Giambattista C, Ventura P, et al. Sex-Gender Comparisons in Comorbidities of Children and Adolescents With High-Functioning Autism Spectrum Disorder. Front Psychiatry 2019;10:159.

16. Westwood H, Tchanturia K. Autism Spectrum Disorder in Anorexia Nervosa: An Updated Literature Review. Curr Psychiatry Rep 2017;19:41.

17. Morris J, Twaddle S. Anorexia nervosa. BMJ 2007;334:894-8.

18. Rask-Andersen M, Olszewski PK, Levine AS, Schiöth HB. Molecular mechanisms underlying anorexia nervosa: focus on human gene association studies and systems controlling food intake. Brain Res Rev 2010;62:147-64.

19. Sauchelli S, Jiménez-Murcia S, Sánchez I, Riesco N, Custal N, Fernández-García JC, et al. Orexin and sleep quality in anorexia nervosa: Clinical relevance and influence on treatment outcome. Psychoneuroendocrinology 2016;65:102-8.

20. Janas-Kozik M, Stachowicz M, Krupka-Matuszczyk I, Szymszal J, Krysta K, Janas A, et al. Plasma levels of leptin and orexin $\mathrm{A}$ in the restrictive type of anorexia nervosa. Regul Pept 2011;168:5-9.

21. Bronsky J, Nedvidkova J, Krasnicanova H, Vesela M, Schmidtova J, Koutek J, et al. Changes of orexin A plasma levels in girls with anorexia nervosa during eight weeks of realimentation. Int $\mathrm{J}$ Eat Disord 2011;44:547-52.

22. Kaye WH, Wierenga CE, Bailer UF, Simmons AN, Bischoff-Grethe A. Nothing tastes as good as skinny feels: the neurobiology of anorexia nervosa. Trends Neurosci 2013;36:110-20.

23. Uniacke B, Timothy Walsh B, Foerde K, Steinglass J. The Role of Habits in Anorexia Nervosa: Where We Are and Where to Go From Here? Curr Psychiatry Rep 2018;20:61.

24. Löwe B, Zipfel S, Buchholz C, Dupont Y, Reas DL, Herzog W. Long-term outcome of anorexia nervosa in a prospective 21-year follow-up study. Psychol Med 2001;31:881-90.

25. Theander S. Outcome and prognosis in anorexia nervosa and bulimia: some results of previous investigations, compared with those of a Swedish long-term study. J Psychiatr Res 1985;19:493508.

26. Hale MD, Logomarsino JV. The use of enteral nutrition in the treatment of eating disorders: a systematic review. Eat Weight Disord 2019;24:179-98.

27. Lock J. Family therapy for eating disorders in youth: current confusions, advances, and new directions. Curr Opin Psychiatry 2018;31:431-5.

28. Grenon R, Carlucci S, Brugnera A, Schwartze D, Hammond N, Ivanova I, et al. Psychotherapy for eating disorders: A meta-analysis of direct comparisons. Psychother Res 2019;29:833-45.

29. Attia E, Steinglass JE, Walsh BT, Wang Y, Wu $\mathrm{P}$, Schreyer $\mathrm{C}$, et al. Olanzapine Versus Placebo in Adult Outpatients With Anorexia Nervosa: A Randomized Clinical Trial. Am J Psychiatry 2019;176:449-56.

30. Dejong H, Broadbent $H$, Schmidt U. A systematic review of dropout from treatment in outpatients with anorexia nervosa. Int J Eat Disord 2012;45:635-47.

31. Channon S, de Silva P, Hemsley D, Perkins R. A controlled trial of cognitive-behavioural and behavioural treatment of anorexia nervosa. Behav Res Ther 1989;27:529-35.

32. Berkman ND, Lohr KN, Bulik CM. Outcomes of eating disorders: a systematic review of the literature. Int J Eat Disord 2007;40:293-309. 
33. Lock J, Le Grange D. Treatment manual for anorexia nervosa: A family-based approach. 2nd ed. New York: Guilford Publications; 2015.

34. Lock J, Le Grange D, Agras WS, Moye A, Bryson SW, Jo B. Randomized clinical trial comparing familybased treatment with adolescent-focused individual therapy for adolescents with anorexia nervosa. Arch Gen Psychiatry 2010;67:1025-32.

35. Robin AL, Siegel PT, Moye AW, Gilroy M, Dennis AB, Sikand A. A controlled comparison of family versus individual therapy for adolescents with anorexia nervosa. J Am Acad Child Adolesc Psychiatry 1999;38:1482-9.

36. Fitzpatrick KK, Moye A, Hoste R, Lock J, le Grange D. Adolescent Focused Psychotherapy for Adolescents with Anorexia Nervosa. Journal of Contemporary Psychotherapy 2010;40:31-9.

37. Serpell L, Treasure J, Teasdale J, Sullivan V. Anorexia nervosa: friend or foe? Int $J$ Eat Disord 1999;25:177-86.

38. Serpell L, Teasdale JD, Troop NA, Treasure J. The development of the P-CAN, a measure to operationalize the pros and cons of anorexia nervosa. Int J Eat Disord 2004;36:416-33.

39. National Institute for Clinical Excellence. Behaviour change at population, community and individual levels. Public Health Guidance. 2007. Available at: http://tinyurl. com/nice-behav-07 [Accessed: January 04, 2011].

40. Abraham C, Michie S. A taxonomy of behavior change techniques used in interventions. Health Psychol 2008;27:379-87.

41. Cuerda C, Vasiloglou MF, Arhip L. Nutritional Management and Outcomes in Malnourished Medical Inpatients: Anorexia Nervosa. J Clin Med 2019;8:1042.

42. Yager J, Andersen A, Devlin M, Egger H, Herzog $\mathrm{D}$, Mitchell $\mathrm{J}$, et al. Practice guideline for the treatment of patients with eating disorders. In: N. C. Numerous Contributors, editors. American Psychiatric Association practice guidelines for the treatment of psychiatric disorders: Compendium. 2nd ed. Washington DC: American Psychiatric Association; 2002. p. 697-766.

43. Mehler PS, Winkelman AB, Andersen DM, Gaudiani JL. Nutritional rehabilitation: practical guidelines for refeeding the anorectic patient. $J$ Nutr Metab 2010;2010:625782.

44. Bargiacchi A, Clarke J, Paulsen A, Leger J. Refeeding in anorexia nervosa. Eur J Pediatr 2019;178:413-22.

45. Stanga Z, Brunner A, Leuenberger M, Grimble RF, Shenkin A, Allison SP, et al. Nutrition in clinical practice-the refeeding syndrome: illustrative cases and guidelines for prevention and treatment. Eur $\mathrm{J}$ Clin Nutr 2008;62:687-94.

46. Leclerc A, Turrini T, Sherwood K, Katzman DK. Evaluation of a nutrition rehabilitation protocol in hospitalized adolescents with restrictive eating disorders. J Adolesc Health 2013;53:585-9.

47. Gentile MG. Enteral nutrition for feeding severely underfed patients with anorexia nervosa. Nutrients 2012;4:1293-303.

48. Diamanti A, Basso MS, Castro M, Bianco G, Ciacco $\mathrm{E}$, Calce $\mathrm{A}$, et al. Clinical efficacy and safety of parenteral nutrition in adolescent girls with anorexia nervosa. J Adolesc Health 2008;42:111-8.

49. Cuerda C, Vasiloglou MF, Arhip L. Nutritional Management and Outcomes in Malnourished Medical Inpatients: Anorexia Nervosa. J Clin Med 2019;8:1042.

50. Takenoya F, Wang L, Kageyama H, Hirako S, Wada $\mathrm{N}$, Hashimoto $\mathrm{H}$, et al. Neuropeptide W-Induced Hypophagia is Mediated Through CorticotropinReleasing Hormone-Containing Neurons. J Mol Neurosci 2015;56:789-98.

51. Takenoya F, Kageyama H, Shiba K, Date Y, Nakazato M, Shioda S. Neuropeptide W: a key player in the homeostatic regulation of feeding and energy metabolism? Ann N Y Acad Sci 2010;1200:162-9.

52. Li H, Feinle-Bisset C, Frisby C, Kentish S, Wittert GA, Page AJ. Gastric neuropeptide $\mathrm{W}$ is regulated by meal-related nutrients. Peptides 2014;62:6-14.

53. Rucinski M, Nowak KW, Chmielewska J, Ziolkowska A, Malendowicz LK. Neuropeptide W exerts a potent suppressive effect on blood leptin and insulin concentrations in the rat. Int $\mathrm{J}$ Mol Med 2007;19:401-5.

54. Kim YR, Kim CH, Park JH, Pyo J, Treasure J. The impact of intranasal oxytocin on attention to social emotional stimuli in patients with anorexia nervosa: a double blind within-subject cross-over experiment. PLoS One 2014;9:e90721. 